\title{
Sociology and bioethics
}

\author{
Bert Gordijn · Wim Dekkers
}

Published online: 18 July 2008

(C) The Author(s) 2008

From a sociological point of view bioethics can be regarded as an intellectual discipline born of mistrust, as the guest editors of this issue's thematic section, Raymond G. De Vries and Scott Kim, argue. Hence they have focused their highly interesting section on "Bioethics and Sociology of Trust". The six studies in this section come from Europe and North America and cover a broad range of issues relevant to the section topic, which will be further introduced by the guest editors in their 'Introduction to the theme'. The rest of this issue consists of four papers.

First, Paula Boddington and Maggie Gregory discuss communication of genetic information in the family. Each year genetic diagnosis and counselling grow more important in clinical practice. They are intended to provide the counselee with information, thus facilitating free and substantiated choices about preventive and therapeutic options. Genetic counselling has been dominated by value-neutrality and non-directiveness as well as autonomy and individual rights. However, the results of genetic tests often reveal information that might have serious implications for the counselee's relatives. In these cases, when strong thirdparty interests are involved, it is increasingly doubted whether the traditional moral precepts might always be adequate. The authors of this paper endeavour to demonstrate that shifting the focus from autonomy to integrity might greatly enrich this debate. In doing so, they draw on theoretical considerations and qualitative empirical data.

B. Gordijn $(\bowtie)$

Nymegen Medical Centre, Radboud University,

Nijmegen, The Netherlands

e-mail: B.Gordijn@iq.umcn.nl

W. Dekkers

Rotterdam University, Rotterdam, The Netherlands
Beatriz Cardona explores 'healthy ageing' policies and anti-ageing ideologies and practices. An ethics of responsibility for health care is being advanced through 'successful ageing' narratives in many Western countries. This approach emphasizes self-constitution and the exercise of the 'responsible self'. Through interviews with anti-ageing consumers, however, it is possible to demonstrate not only the tensions and contradictions, which such a rigid model of self-constitution in later life produces, but also the potential forms of resistance and contestations that may emerge as a result.

The next paper, by Julian C. Hughes, Claire Bamford and Carl May, focuses on different types of centredness in health care, such as client-, family-, patient-, person- and relationship-centred care. Selected reviews and papers about this topic were analysed as text transcripts and subjected to a philosophical analysis using notions from Wittgenstein's philosophy. The authors conclude that whilst practical utility justifies different types of centredness in different contexts, the unifying themes of centredness reflect a movement promoting the understanding of the social, psychological, cultural and ethical dimensions of our human encounters.

Finally, Georg Spielthenner analyses the principle of double effect as a guide for decision-making in medicine. Double-effect reasoning is often referred to in situations when health care professionals are not able to accomplish a benefit without bringing about some harm. Although the principle certainly has its advantages, the author concludes that, on the whole, it does not provide physicians and nurses with sufficient ethical guidance in clinical practice.

Open Access This article is distributed under the terms of the Creative Commons Attribution Noncommercial License which permits any noncommercial use, distribution, and reproduction in any medium, provided the original author(s) and source are credited. 